Notfall Rettungsmed 2016 · 19:54-55

DOI 10.1007/s10049-015-0124-7

Online publiziert: 1. Februar 2016

(c) Springer-Verlag Berlin Heidelberg 2016

CrossMark

G. Monsieurs ${ }^{1,2} \cdot$ J.P. Nolan ${ }^{3} \cdot$ L.L. Bossaert ${ }^{4,5} \cdot$ R. Greif ${ }^{6} \cdot$ I.K. Maconochie ${ }^{7}$.

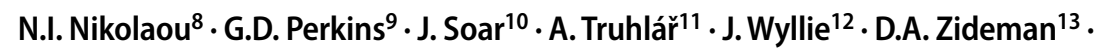
on behalf of the ERC Guidelines 2015 Writing Group

${ }^{1}$ Emergency Medicine, Faculty of Medicine and Health Sciences, University of Antwerp, Antwerp, Belgien

${ }^{2}$ Faculty of Medicine and Health Sciences, University of Ghent, Ghent, Belgien

${ }^{3}$ Anaesthesia and Intensive Care Medicine, Royal United Hospital, Bath, UK

${ }^{4}$ Bristol University, Bristol, UK

${ }^{5}$ University of Antwerp, Antwerp, Belgien

${ }^{6}$ Department of Anaesthesiology and Pain Medicine, University

Hospital Bern and University of Bern, Bern, Schweiz

${ }^{7}$ Paediatric Emergency Medicine Department, Imperial College Healthcare

NHS Trust and BRC Imperial NIHR, Imperial College, London, UK

${ }^{8}$ Cardiology Department, Konstantopouleio General Hospital, Athens, Griechenland

${ }^{9}$ Warwick Medical School, University of Warwick, Coventry, UK

${ }^{10}$ Anaesthesia and Intensive Care Medicine, Southmead Hospital, Bristol, UK

${ }^{11}$ Emergency Medical Services of the Hradec Kralove Region and Department of Anaesthesiology and Intensive Care Medicine, University Hospital Hradec Kralove, Hradec Kralove, Tschechien

${ }^{12}$ Department of Neonatology, The James Cook University Hospital, Middlesbrough, UK

${ }^{13}$ Imperial College Healthcare NHS Trust, London, UK

\title{
Erratum zu: Kurzdarstellung. Kapitel 1 der Leitlinien zur Reanimation 2015 des European Resuscitation Council
}

In $\bullet$ Abb. 16 ist ein Fehler unterlaufen. In der unteren blauen Box auf der rechten Seite wird eine schlechte neurologische Erholung als „sehr wahrscheinlich“ angegeben. Die richtige Angabe ist jedoch „wahrscheinlich“. Bitte beachten Sie die hier dargestellte, korrigierte $\bullet$ Abb. 16.

\section{Korrespondenzadresse}

\section{G. Monsieurs}

Emergency Medicine,

Faculty of Medicine and Health Sciences

University of Antwerp, Antwerp

dirks@grc-org.de

Korrespondierender Übersetzer

Dr. rer. nat. Dr. med. Burkhard Dirks Badbergstrase 18, 89075 Ulm dirks@grc-org.de 


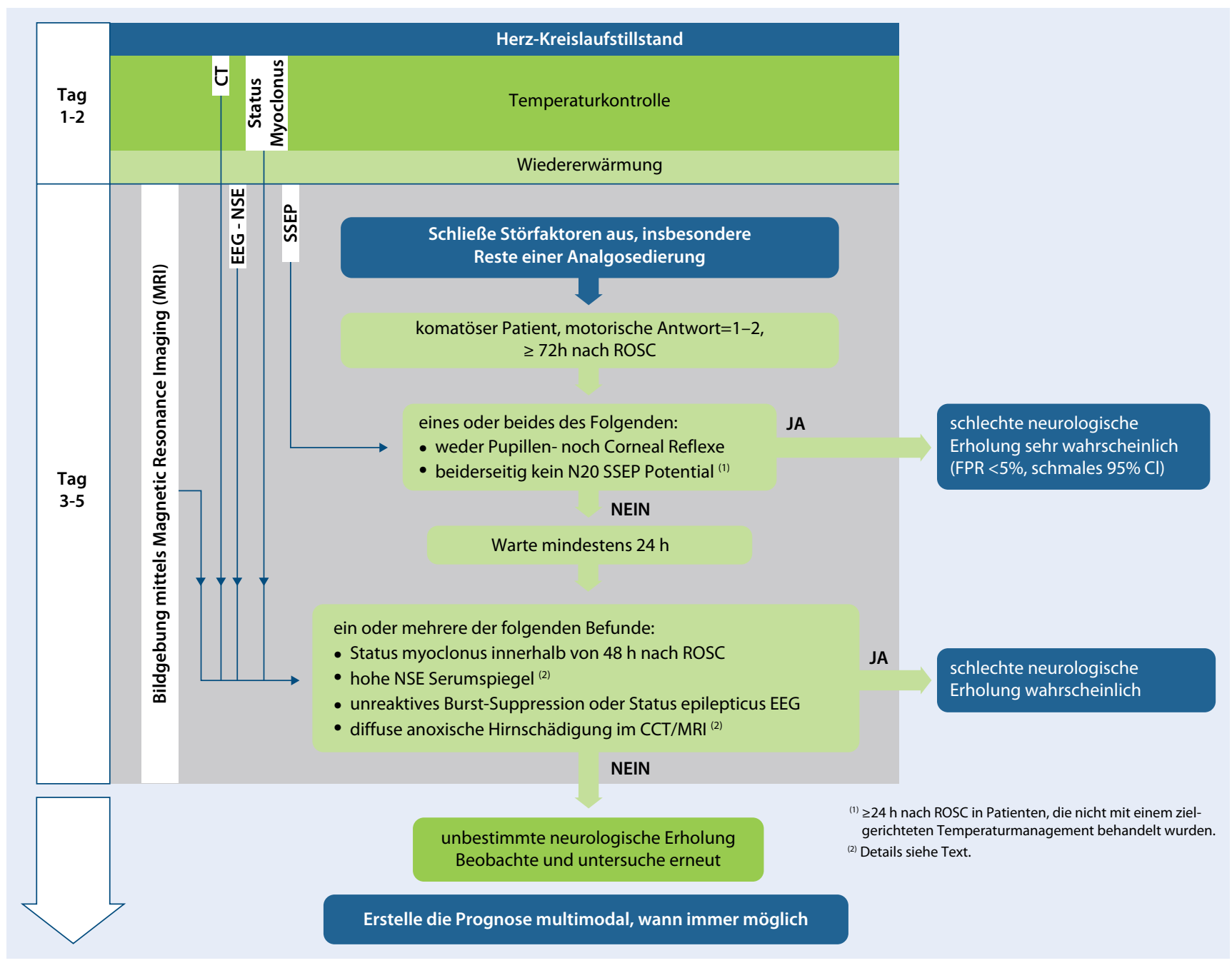

Abb. 16 ॥ Algorithmus zur Prognoseerstellung. EEG Elektroenzephalogramm; NSE neuronenspezifische Enolase; SSEP somatosensorische evozierte Potenziale; ROSC Rückkehr des Spontankreislaufs 